\title{
Modal Superlatives
}

\author{
Bernhard Schwarz \\ McGill University
}

\section{Non-local Modification with Possible}

Larson (2000) reports that prenominal possible can semantically compose with its syntactic context in two fundamentally different ways. In ordinary cases, possible combines locally with a property-denoting common noun phrase into another property-denoting common noun phrase. The strings possible liar and possible textbook in (1)a and (1)b, for example, are common noun phrases denoting the property of possibly being a liar and the property of being possible as a textbook, respectively.

(1) a. We are dealing with a possible liar.

b. They sent me a possible textbook.

But Larson suggests that semantic composition with prenominal possible is not always local in this way. For example, sentence (2) is unlikely to convey that I attended every event that was possibly a class or that I attended every event that was possible as a class.

(2) I attended every possible class.

The actual reading of sentence (2) might be paraphrased as in (3), where possible does not locally combine with class into a property-denoting common noun phrase, but instead is part of a restrictive relative clause modifying class.

(3) I attended every class [that it was possible for me to attend].

Given paraphrases like (3), Larson refers to the reading illustrated by (2) as "implicit relative reading". Below I will use the term "non-local reading" instead.

Larson observes that non-local modification with prenominal possible is found only in universal and superlative noun phrases. Non-local possible in universals is illustrated in (2) above. Sentence (4)a, in the reading indicated in (4)b, illustrates non-local possible in superlatives. I will refer to such cases as "modal superlatives".

a. I bought the largest possible present.

b. I bought the largest present [that it was possible for me to buy].

The cases in (2) and (4)a contrast with those in (1) and all versions of the examples in (5), which lack non-local readings. To the extent that the examples in (5) are acceptable, possible only has a local interpretation. 
(5) a. I took the/a/some/at least one possible class.

b. I took two/many/several/most possible classes.

Larson proposes that the relative clause paraphrases of non-local readings with prenominal possible point to the proper analysis of such readings. Specifically, he proposes that non-local prenominal possible originates in a postnominal position, where it is part of a partially elided reduced relative clause.

This paper argues that non-local prenominal possible in superlatives should not be so analyzed. Based on several new observations, it is proposed instead that in these cases non-local readings arise because adjacent superlative est and possible can form a modal degree operator that takes non-local scope.

\section{The Implicit Relative Analysis}

Larson reports that possible can also appear in postnominal position. This is illustrated in (6). Larson moreover notes that postnominal possible is unambiguously non-local. Postnominal possible is then expected to be unacceptable in noun phrases that are known not to support non-local readings. This expectation is correct. The examples in (7), which contrast minimally with those in (5), are unacceptable.

(6) a. I attended every class possible.

b. I bought the largest present possible.

(7) a.* I took the/a/some/at least one class possible.

b.* I took two/many/several/most classes possible.

Larson further observes that in addition to paraphrases where possible appears in a finite relative clause, non-local readings have paraphrases with a reduced relative clause. ${ }^{1}$ The non-finite postnominal modifiers in (8) seem synonymous with those in (3) and (4)b, and hence with those in (6).

a. I attended every class [possible for me to attend].

b. I bought the largest present [possible for me to buy].

These observations lead Larson to propose that prenominal non-local possible originates in a postnominal reduced relative clause, with the relevant surface forms being derived by syntactic promotion of possible to a prenominal position and by ellipsis of its stranded infinitival complement. The resulting surface structures for (2) and (4)a are sketched in (9).

(9) a. I attended every [possible $e_{2}$ class $w_{1} \mathrm{e}_{2}$ [for to atend $\mathrm{e}_{1}$ ]

b. I bought the largest [possible present $\mathrm{wh}_{1} \mathrm{e}_{2}$ [for me-to-buy $\mathrm{e}_{1}$ ]]. 
Larson notes that the ellipsis posited in this analysis is antecedentcontained, much like better-known antecedent-contained verb phrases illustrated in (10).

I attended every class [I could].

He suggests that antecedent-containment is resolved in the same way in both types of ellipsis. Specifically, Larson adopts the standard account of Sag (1976) and May (1985), which assumes that in antecedent-contained ellipsis, a proper ellipsis antecedent is created at logical form by extraction of the noun phrase containing the ellipsis site from the antecedent. In this account, then, the logical forms of (2) and (4)a are as shown in (11). ${ }^{2}$

(11) a. [every class $w h_{1}$ possible for to $\left.e_{1}\right]_{3}\left[I\right.$ attended $e_{3}$ ]

b. [the largest present $w_{1}$ possible $\left.e_{1}\right]_{3}\left[I\right.$ bought $\left.e_{3}\right]$

Before starting to question the implicit relative analysis for modal superlatives in the next section, let me propose a possible amendment. Under the implicit relative analysis the examples in (6) indicate that it is possible for ellipsis to apply without promotion of possible. Indeed, the null assumption should be that ellipsis and promotion can apply independently of each other. However, it appears that even though there can be ellipsis without promotion as in (6), promotion is dependent on the application of ellipsis. The sentences in (12), for instance, do not have non-local interpretations.

(12) a. I attended every possible class [for me to attend].

b. I bought the largest possible present [for me to buy].

To the extent that these cases are acceptable, possible only receives a local interpretation and the postnominal expressions are parsed as infinitival relative clauses.

A slight modification of Larson's analysis may be sufficient to account for this restriction on promotion. Suppose promotion never targets the adjectival head possible itself, but instead targets the phrase comprising the head and its clausal complement. The structures in (9) are then to be replaced with those in (13). ${ }^{3}$

a. I attended every [ [possible $\left.\mathrm{e}_{1}\right]_{2}$ class $\mathrm{wh}_{1} \mathrm{e}_{2}$ ].

b. I bought the largest [ [possible for to buy $\left.\mathrm{e}_{1}\right]_{2}$ present $\mathrm{wh}_{1} \mathrm{e}_{2}$ ].

If promotion is phrasal movement, then promotion can never strand an overt infinitival complement clause in postnominal position and the lack of non-local readings in (12) follows.

The remaining question is whether structures like those in (13) are indeed well-formed. After all, it is known that prenominal adjectives in English cannot usually be overtly separated by a complement from the common noun phrase they modify. Strings like the proud of his son father or the eager to leave guests are 
not acceptable noun phrases. More to the point, the strings in (13) themselves with the crossed out material pronounced are not acceptable sentences. For the parses in (13) to be licit, therefore, it must be assumed that the constraint in question is a constraint on phonetic form rather than a constraint on more abstract surface forms. Under this assumption, ellipsis of the infinitival complement of possible can rescue an otherwise illicit phonetic form.

The implicit relative analysis is an attractive account of non-local readings with interesting implications for the theory of ellipsis and the syntax of noun phrases. However, the next section will present data from both German and English that cast some doubt on the correctness of this account as it applies to modal superlatives.

\section{Questions about Modal Superlatives}

\subsection{Modal Superlatives in German}

English universals with non-local possible do not have direct counterparts in German. For example, I cannot naturally use (14) in the same way as its English translation, conveying that I registered for every course I could. But as (15) illustrates, möglich can be interpreted non-locally in superlative noun phrases.

(14) Ich habe jeden möglich.en Kurs belegt.

I have every possible.Infl course registered

'I registered for every possible course.'

(15) Ich habe das größt möglich.e Geschenk gekauft.

I have the largest possible.Infl present bought

'I bought the largest present possible.'

German modal superlatives are of particular interest because they are morphologically peculiar. Prenominal adjectives in German usually carry one of the suffixes -er, $-e$, -es, or -en, depending on the gender, number, and case of the common noun and on the definiteness of the containing noun phrase. Superlative adjective like größt in (15) above can exceptionally lack such an agreement suffix. In one possible description of the phenomenon, möglich and the superlative adjective "share" one agreement inflection.

Möglich is exceptional in its ability to share agreement morphology with a superlative adjective. This is illustrated in (16), where grö $\beta t$ 'largest' is followed by the ordinary modifier bezahlbar 'affordable' and cannot remain uninflected.

(16) Ich habe das größt.*(e) bezahlbar.e Geschenk gekauft. I have the largest.Infl affordable.Infl present bought

'I bought the largest affordable present.' 
There is a clear correlation between inflection sharing and interpretation. Shared inflection forces a non-local interpretation of möglich whereas independent inflection excludes it. As the translation indicates, (15) only has a non-local reading. Conversely, example (17), where größte carries its own inflection, only permits a local interpretation of möglich.

Ich habe das größt.e möglich.e Geschenk gekauft.

I have the largest.Infl possible.Infl present bought

'I bought the largest of the possible presents.'

According to the description given in Corver (1997), Dutch mogelijk 'possible' licenses much the same kind of agreement sharing in superlatives described here for German möglich. Corver notes that agreement sharing is associated with an unusual semantics. He does not actually characterize the relevant readings in detail, but it seems reasonable to suspect that they are analogous to the non-local interpretations described here for English and German.

The German facts presented here raise the question whether in English, non-local possible in universals and non-local possible in superlatives really are the same kind of phenomenon. The German data also cast some doubt on the implicit relative analysis for modal superlatives. This is because possible source structures analogous to the English examples in (6) and (8) are not attested in German. In particular, möglich cannot appear on its own in postnominal position. Finally, the implicit relative account does not by itself make sense of the correlation between non-local interpretation and agreement sharing.

Of course, the German facts as such do not present a strong argument against an implicit relative analysis for the English cases. It is certainly conceivable that distinct mechanisms underlie what superficially looks like the same phenomenon in the two languages. However, the next subsection will show that even confining attention to English, an implicit relative analysis for modal superlatives can be questioned, as it needs to attribute properties to promotion that seem to lack independent motivation.

\subsection{Constraints on Modal Superlatives in English}

As Larson (2000) observes, non-local possible in universals must be right adjacent to the universal determiner. Sentence (18) for example does not have a non-local interpretation. In the implicit relative analysis, this would seem to indicate that promotion targets a dedicated position that precedes all other prenominal modifiers.

I attended every introductory possible class.

However, modal superlatives, where the superlative adjective intervenes between the determiner and possible, indicate that promotion is not in general so constrained. As illustrated by the observation that (19) lacks a non-local reading, 
possible in modal superlatives instead must be right adjacent to the superlative adjective.

(19) I bought the largest affordable possible present.

The obvious question is how to independently motivate a theory of promotion that is able to reconcile the word order required in superlatives with the word order required in universals.

More questions for the theory of promotion arise from two restrictions for modal superlatives not previously described. One restriction is that prenominal possible cannot be interpreted non-locally in analytic superlatives, that is, in superlatives formed with most. To illustrate, the examples in (20) only have local interpretations. That is, sentence (20)a cannot be read as saying that I bought the most expensive present I could and (20)b cannot convey that she rented the most luxurious car she could.

(20) a. I bought the most expensive possible present.

b. She rented the most luxurious possible car.

The examples in (20) contrast minimally with those in (21), which do have these non-local interpretations. (In fact, in accordance with the Larson's generalization on postnominal possible, these examples cannot be understood locally.)

(21) a. I bought the most expensive present possible.

b. She rented the most luxurious car possible.

Evidently, given the acceptability of these variants, the absence of non-local readings in (20) cannot be credited to a general constraint against non-local readings in analytic superlatives. Instead, a implicit relative analysis will have to specifically attribute the judgments on (20) to a constraint on promotion.

A pattern similar to that attested with analytic superlatives is found with complex superlative adjectives such as fastest-growing or fastest-paced, where an adjective with synthetic superlative morphology modifies a following present or past participle. Such modified participles are usually interpreted much like ordinary superlative adjectives. However, they do not allow for a non-local interpretation of a following possible. This is illustrated in (22). Only local readings are available for these examples; sentence (22)a cannot mean that I bought the fastest-growing plant I could, nor can (22)b mean that we play the fastest-paced game we can.

(22) a. I bought the fastest-growing possible plant.

b. We play the fastest-paced possible game.

As with analytic superlatives, this is a restriction on modal superlatives with prenominal possible and not a restriction on modal superlatives in general. The 
variants of the cases in (22) shown in (23) do have non-local readings. (And again, as expected, these examples do not in fact have local interpretations.)

(23) a. I bought the fastest-growing plant possible.

b. We play the fastest-paced game possible.

In a implicit relative analysis, therefore, the unavailability of non-local readings in (22) presumably is again indicative of a constraint on promotion.

The question is of course how a constraint of the sort needed here can be formulated and independently motivated. The descriptive generalization that emerges is that prenominal possible can have a non-local interpretation only if it immediately follows a superlative adjective ending in the superlative suffix est. Assuming this generalization, the implicit relative analysis of modal superlatives will thus need to assume that this is the only prenominal position to which possible can be promoted. It is unclear how a constraint with this effect would fit into a general theory of syntactic movement.

In conjunction with the German agreement facts, the constraints on nonlocal readings in English presented here therefore cast doubt on an implicit relative analysis for modal superlatives with prenominal possible and so they provide some incentive to search for an alternative. Such an alternative analysis is presented in the next section.

\section{A Modal Degree Operator}

\subsection{The Syntax of Modal Superlatives}

As mentioned above, the kind of agreement sharing phenomenon found in German is already described in Corver (1997) for Dutch. Corver makes the plausible assumption that in order for two adjectives to share an agreement inflection, they must form a constituent. Corver also assumes that the relevant structures for English and Dutch are parallel, the main difference being that Dutch but not English adjectives carry overt agreement morphology. Example (4)a above would accordingly be parsed as in (24).

(24) I bought the [largest possible] present.

Corver more specifically proposes that possible in modal superlatives is an "auxiliary adjective" that takes the superlative adjective phrase as its complement. Corver also follows Abney (1987) in the assumption that AP is a complement of a Deg head, which he considers a landing site for superlative adjectives. Thus a superlative adjective like largest would have the underlying structure in (25)a and head movement would string vacuously take largest to Deg. To the string largest possible in modal superlatives Corver accordingly assigns the underlying structure in (25)b. Head movement of the superlative adjective into the Deg head establishes the actually observed word order. 

a. $\left[{ }_{\text {DegP }}\left[{ }_{\text {Deg }}\right]\left[\right.\right.$ APP $_{\text {large+est }]]}$
b. [DegP $[\mathrm{Deg}]\left[\mathrm{AP}[\mathrm{A}\right.$ possible $]\left[{ }_{\mathrm{AP}}[\mathrm{A}\right.$ large + est $\left.\left.\left.]\right]\right]\right]$

Corver derives agreement sharing in analogous structures for Dutch from the assumption that the auxiliary adjective is the only carrier of inflection in its $A P$, just like an auxiliary verb is the only finite verb in its VP.

While this analysis makes sense of agreement sharing in Dutch and German modal superlatives, it is less clear whether it is able to accommodate the restrictions on the form of modal superlatives in English identified above. In particular, it is not clear to me that the underlying structures in (26) could not incorrectly derive modal superlative parses for the strings most expensive possible and fastest-growing possible, respectively.

$$
\begin{aligned}
& \text { a. }\left[{ }_{\text {DegP }}[\operatorname{Deg} \operatorname{most}]\left[{ }_{A P}[A \text { possible }]\left[{ }_{A P}\left[{ }_{A} \text { expensive }\right]\right]\right]\right] \\
& \text { b. }\left[{ }_{\text {DegP }}\left[{ }_{\text {Deg }}\right]\left[{ }_{A P}[A \text { possible }]\left[{ }_{A P}[A \text { fast+est-growing }]\right]\right]\right]
\end{aligned}
$$

More importantly, the assumption that possibility adjectives can share agreement inflection with their adjectival complements does not seem to help derive the characteristic non-local readings of modal superlatives. Under the parse in (25)b, one might perhaps expect the relevant reading of (4)a to convey that I bought a present that is possibly the largest. The sentence does not have such an interpretation and it is hard to see how (25)b might derive the intended non-local reading conveying that $I$ bought the largest present I could.

In the following I will therefore explore an alternative structure for modal superlatives. I will maintain Corver's assumption that in modal superlatives the modal adjective and the superlative form a constituent. However, I proposes a different internal structure for this constituent. I suggest that the immediate constituents of the modal superlative largest possible are not largest and possible, but rather large and est possible; I suggest est possible is a degree operator with the same categorial status as bare est.

Preparing for the semantic analysis presented in the next section, I moreover replace the internal structure of graded adjective phrases proposed in Abney (1987) and Corver (1997), with the more traditional structure assumed in works like Bresnan (1973) and Heim (2000): Instead of construing AP as a complement of Deg, I will take DegP to occupy the specifier position of A. In this view, the modal superlative largest possible is derived from (27)b in the same way that bare largest is derived from (27)a. That is, just like bare est, the modal degree operator est possible must be pronounced as a suffix on the following adjectival head.
a. [ ${ }_{A P}\left[{ }_{\text {eegP }}\right.$ est $]\left[{ }_{A}\right.$, large] ]
b. $\left[_{A P}\left[D_{\text {egP }}\right.\right.$ est possible $]\left[{ }_{A}\right.$, large $\left.]\right]$

In analogy to the surface parse gros $+s t+e$ for the ordinary German superlative größte 'largest', then, the modal superlative größt mögliche is now parsed as gros + [st möglich] $+e$. This parse for modal superlatives posits only one 
adjective that might carry agreement inflection in a modification structure and so it straightforwardly predicts the agreement sharing facts in German and Dutch. Notice also that this analysis differs from Corver's in that it identifies the superlative adjective as the carrier of the inflection, rather than the possibility adjective.

The restrictions on English modal superlatives described above now also receive a straightforward explanation. The examples in (19), (20), and (21), repeated below, are predicted to lack non-local interpretations for the simple reason that they cannot be parsed as containing the modal degree operator est possible. $^{4}$

(19) I bought the largest affordable possible present.

(20) a. I bought the most expensive possible present.

b. She rented the most luxurious possible car.

(21) a. I bought the fastest-growing possible plant.

b. We play the fastest-paced possible game.

The remaining task is to demonstrate that this new parse for modal superlatives provides a basis for deriving non-local readings of the sort described in Larson (2000). This task is taken up in the next subsection.

\subsection{Interpreting Modal Superlatives}

In this subsection I propose that the non-local interpretations of modal superlatives can be credited to logical forms similar to the logical forms posited in Heim (1999) for so-called comparative readings of ordinary superlative noun phrases.

Heim (1985) and Szabolcsi (1986) observe that superlative noun phrases are often ambiguous between an "absolute" and a "comparative" reading. For example, in its absolute reading sentence (28) conveys that John climbed the highest of all mountains, while in the comparative reading it merely asserts that John climbed a higher mountain than any other relevant person did.

(28) John climbed the highest mountain.

Heim (1999) shows how this ambiguity can be analyzed as a scope ambiguity. ${ }^{5}$ In Heim's analysis, the absolute reading results when est takes scope locally within its noun phrase. The comparative reading, which is the reading I will focus on here, is credited instead to a logical form where est takes non-local scope over the entire clause. Heim moreover assumes that when est scopes outside its noun phrase, the definite article exceptionally has the semantics of indefinite $a$. To illustrate, in this analysis the comparative reading of (28) is due to the logical form in (29). 


$$
\text { [DegP } \text { est ] } \lambda_{1}\left[\text { John climbed A } \left[_{A P} e_{1}\right.\right. \text { high] mountain] }
$$

In (29), est has covertly extracted from its noun phrase to the edged of the clause and leaves behind the trace $e_{1}$, a variable ranging over degrees. The lambda abstract to the right of est accordingly denotes a property of degrees, that is, a function from degrees to truth values. Which property of degrees? As indicated, the definite article the is taken to be interpreted like its indefinite counterpart $a$. Heim furthermore takes gradable adjectives to relate individuals and degrees and assigns them an "at least" semantics. The denotation of $\left[_{A P}[3\right.$ meters] high], for example, is taken to characterize the set of individuals that are at least 3 meters high. Accordingly, the lambda abstract in (29) characterizes the set of degrees $d$ such that John climbed a mountain that is at least $\mathrm{d}$ high.

The last piece needed to interpret the logical form in (29) is the denotation of $e s t$ itself. Heim proposes a lexical entry much like (30). There, d ranges over degrees and $\mathrm{P}$ and $\mathrm{Q}$ range over properties of degrees; $\mathrm{C}$ is a contextually determined set of properties of degrees.

$$
[[\text { est }]]=\lambda \mathrm{P} . \exists \mathrm{d}[\mathrm{P}(\mathrm{d})=1 \& \forall \mathrm{Q}[\mathrm{Q} \in \mathrm{C} \& \mathrm{Q} \neq \mathrm{P} \rightarrow \mathrm{Q}(\mathrm{d})=0]]
$$

According to (30), then, a logical form est $\phi$ is true just in case for some degree $\mathrm{d}$ the property of degrees denoted by $\phi$ is the only property in $\mathrm{C}$ that $\mathrm{d}$ has. With the appropriate choice for $C$ this yields the intended comparative reading for (29). Suppose $C$ is the set of degree properties $\{\lambda d . x$ climbed an at least $d$ high mountain: $x$ is a relevant person\}. Then (29) comes out as true just in case there is a degree such that John is the only relevant person who climbed a mountain that is high to at least that degree, that is, just in case John climbed a higher mountain than any other relevant person. ${ }^{6}$

Assuming the structure for modal superlatives proposed above, I suggest that their interpretations can be credited to logical forms isomorphic to those that Heim (1999) assumes for comparative superlatives. For example, I propose that, assuming a suitable lexical entry for est possible, the non-local interpretation of sentence (4)a above can be derived from the logical form in (31).

$$
\text { [DegP } \text { est possible] } \lambda_{1}\left[\mathrm { I } \text { bought } \mathrm { A } \left[{ }_{A P} \mathrm{e}_{1}\right.\right. \text { large] present ] }
$$

The lexical entry for est possible that is needed here is shown in (32). Here, $\mathrm{d}$ again ranges over degrees; $\mathrm{P}$ ranges over intensional degree properties, that is, functions from possible worlds to properties of degrees; $w$ and w' range over possible worlds; and $\mathrm{R}$ is an accessibility relation between possible worlds. Also, the lexical entry is relativized to a world parameter, which in unembedded contexts will be set to the actual world.

$$
[[\text { est possible }]]^{\mathrm{w}}=\lambda \mathrm{P} . \forall \mathrm{d}\left[\exists \mathrm{w}^{\prime}\left[\mathrm{wRw}^{\prime} \& \mathrm{P}\left(\mathrm{w}^{\prime}\right)(\mathrm{d})=1\right] \rightarrow \mathrm{P}(\mathrm{w})(\mathrm{d})=1\right]
$$

According to (32), a logical form [est posssible] $\phi$ is true just in case in the actual world the degree property denoted by $\phi$ holds of any degree that it holds of in any 
accessible world. Specifically, the logical form in (31) comes out true just in case for any degree $d$, if in some accessible world I bought a present that is at least $d$ large, then in the actual world, too, I bought a present that is at least $\mathrm{d}$ large. More colloquially, (31) comes out true just in case I bought as large a present as possible. I submit that this is the intended non-local reading.

To summarize, the analysis of modal superlatives presented here derives non-local readings for modal superlatives by positing a modal degree operator and logical forms analogous to those for comparative readings in the analysis of Heim (1999). The analysis is motivated by agreement sharing facts in German and related restrictions on modal superlatives in English, which remain unexplained in the implicit relative analysis.

Before the modal degree operator analysis can be considered a success, however, its consequences need to be studied in greater detail and many questions need to be given satisfactory answers. The next section contains a brief and inconclusive examination of some of the relevant issues.

\section{Consequences, Problems, Speculations}

\subsection{Non-Compositionality}

The analysis offered here stipulates a denotation for the modal degree operator est possible. Ideally, of course, this denotation can be derived compositionally from independently motivated denotations of est and possible through familiar principles of semantic composition. Unfortunately, however, such a derivation is unlikely to succeed.

Let us compare the lexical entries in (30) and (32). Note first that according to these lexical entries, est and est possible compare different types of objects. While est compares the denotation of its sister constituent with other degree properties, est possible compares the actual world with other possible worlds.

Naturally, in a compositional derivation, the fact that est possible compares possible worlds would need to be credited to the semantic contribution of possible. I will leave open, though, the question whether this can be done in a principled way. For even if the answer is yes, a compositional derivation of the intended denotation for est possible is still unlikely. This is because according to (30) and (32), the comparison expressed by est possible has a different "force" than the comparison expressed by bare est.

In a schematic rendering of (30), est $\phi$ states that the degree property denoted by $\phi$ ranks higher than any of its alternatives. In an equally schematic rendering of (32), in contrast, [est posssible] $\phi$ states that the actual world ranks at least as high as any of its alternatives. So while est according to (30) has the force of a comparative, est possible according to (32) instead has the force of an equative; while (30) renders est equivalent to an instance of er ... than ..., (32) makes est possible equivalent to an instance of (at.least) as ... as ... . 
This suggests that est in the modal degree operator est possible cannot be assigned the same semantics as bare est and hence that the denotation in (32) cannot be compositionally derived.

This non-compositionality of est possible can certainly be held against the modal degree operator analysis proposed here. One possible defense would consist in the demonstration that superlative morphology goes with an equative semantics in other cases as well, and so that the apparent non-compositionality is a puzzle that exists independently of the proposal made here. In fact, there are data that might be so interpreted. For example, the synonymy of (33)a and (33)b might be taken to indicate that the adverbial the best I could, while morphologically superlative, has equative rather than comparative force.

a. I did my job the best I could.

b. I did my job as well as I could.

But while examples of this sort may be suggestive, they of course do not by themselves present independent evidence for the view that est can have an equative semantics. Naturally, a complete argument to this effect would need to be based on a detailed analysis of adverbials of the kind featured in (33). I will not attempt such a detailed analysis here and so I will leave open the question whether such examples can indeed be used to defend non-compositionality in the analysis proposed here.

As matters stand, therefore, non-compositionality is an unattractive feature of the present account that needs to be weighed against the empirical advantages of the analysis.

\subsection{Uniqueness Implications}

The reader will have noticed that the non-local interpretations derived in the present account are not exactly those derived in Larson's (2000) implicit relative clause analysis. Consider (34) in its non-local reading.

I climbed the highest possible mountain.

In the implicit relative analysis, this non-local reading is due to the logical form sketched in (35). This logical form comes out true just in case I climbed the highest of all those mountains that it was possible for me to climb and hence implies that there is a unique highest mountain among those that it was possible for me to climb.

[the highest [mountain $w h_{1}$ possible $\left.\left.e_{1}\right]\right]_{3}\left[I\right.$ climbed $e_{3}$ ]

This uniqueness implication is contributed twice in (35), once by superlative est and once by the definite article the.. Neither of these two sources of uniqueness is present in (36) below, the logical form that derives the non-local 
reading of (34) in the present account. By assumption, in this logical form the definite article is replaced with its indefinite counterpart. Est possible does not contribute uniqueness either, as it has been assigned equative rather than comparative force, making (36) true just in case I climbed as high a mountain as possible

$$
\text { [DegP } \text { est possible] } \lambda_{1}\left[\text { I climbed } A \left[{ }_{A P} e_{1}\right.\right. \text { high] mountain] }
$$

In contrast to the logical form (35), then, (36) can be true in a scenario where two mountains of the same height are the highest actual mountains I could have climbed.

So there is a clear difference in predictions between the implicit relative analysis and the modal degree operator analysis. Only the latter predicts the nonlocal reading of (34) to be true in, say, the following scenario: I am able to climb any mountain that is up to $2000 \mathrm{~m}$ high but no mountain higher than that; there are two actual $2000 \mathrm{~m}$ mountains; I climbed one of the two.

Some speakers indeed seem to perceive weaker uniqueness implications in modal superlatives than in ordinary superlatives. They are willing to judge the non-local reading of sentence (34) true in the scenario just described, in contrast to the ordinary superlative version in (37), which they refuse to judge true in the same situation.

I climbed the highest of the mountains that I could climb.

However, these speakers also report that the above scenario is more naturally described by sentence (38) than by sentence (34) itself. This contrast is not expected under the modal degree operator analysis, according to which (38) should be a perfect paraphrase of (34).

I climbed as high a mountain as I could.

It appears, then, that neither of the two analyses under consideration completely succeeds in accounting for uniqueness judgments. A closer examination of uniqueness implications is a task for future research.

\subsection{Restrictions on Covert Movement}

In the modal degree operator analysis, non-local readings of modal superlatives are due to logical forms isomorphic to the logical forms that Heim (1999) posits for comparative superlatives. In both cases, the degree operator extracts from the superlative noun phrase that contains it at the surface and takes clausal scope. This covert scoping of degree operators is expected to be subject to syntactic restrictions. Moreover, whatever restrictions there are on scoping of est should also be restrictions on the scoping of est possible; non-local readings and comparative readings should be subject to the same syntactic restrictions. 
There are observations that seem to bear out this expectation. One constraint on comparative readings (not described in previous literature) is that they are unavailable with superlative noun phrases introduced by a prenominal Saxon genitive. For example, (39) only has an absolute reading according to which he read the paper of mine that is longer than all the other papers of mine. The sentence cannot be understood as saying that he read a longer paper of mine than any other relevant person did.

He read my longest paper.

In Heim's analysis of comparative readings, the absence of the comparative reading in (39) indicates that prenominal genitives block extraction of the degree operator from the containing noun phrase. ${ }^{8}$

I do not know why prenominal genitives should block extraction of est. However, assuming that they do, the present analysis predicts that prenominal genitives also exclude non-local readings of modal superlatives. This prediction is borne out. Sentence (40), for example, only has the somewhat unnatural local reading according to which he read the longest of my possible papers.

(40) He read my longest possible paper.

The observation that both non-local readings of modal superlatives and comparative readings of ordinary superlatives are blocked by prenominal genitives is welcome under the modal degree operator analysis. However, it must be noted that non-local readings with prenominal genitives are also unavailable in cases where possible appears postnominally. Consider the variant of (40) in (41). This example does not have local interpretation, as postnominal possible can never be so interpreted; and the fact that it is unacceptable indicates a non-local reading is not available either.

?? He read my longest paper possible.

It is unclear why (41) should lack a non-local interpretation. But whatever explanation might be given for this fact, the implicit relative analysis can presumably explain the absence of this reading in (40) in the same way. Therefore, examples like (40) do not in fact favor the modal degree operator analysis over an implicit relative analysis.

There is another type of case, however, which does seem to distinguish the two accounts. Superlative noun phrases where the superlative adjective is preceded by the intensifier very do not seem to have comparative readings. Thus it seems that (42) cannot mean that we bought a finer wine than everyone else did. The sentence only has an absolute reading, according to which we bought wine that is finer than all other wines.

We bought the very finest wine. 
This suggests that est cannot covertly move past very and so the modal degree operator analysis predicts that est possible cannot, either. It is predicted then, that noun phrases with very also do not permit non-local readings of modal superlatives with prenominal possible. This seems correct, as it appears that (43) below only has a (rather unnatural) local interpretation, according to which we bought the very finest among the possible wines.

(43) We bought the very finest possible wine.

In the implicit relative analysis, in contrast, the unavailability of a nonlocal reading in (43) is unexpected. For this time such a non-local reading is attested in the corresponding example with postnominal possible. The example in (44), which differs from (43) only in the position of possible, seems fully acceptable.

(44) We bought the very finest wine possible.

In summary, under the modal degree operator analysis the observation that comparative readings and non-local readings are similarly constrained by the syntactic form of the superlative noun phrase naturally follows from the assumption that covert movement of est and covert movement of est possible are subject to the same constraints. In the implicit relative analysis, in contrast, the observed match between constraints on comparative and non-local readings appears accidental.

\subsection{Postnominal Possible}

An obvious open issue is the analysis of superlatives with postnominal non-local possible. The main virtue of Larson's implicit relative analysis is that it explains in a straightforward way the equivalence of postnominal possible and prenominal non-local possible. As it stands, the present analysis leaves this equivalence unaccounted for. While I will not attempt to actually close this gap here, I will suggest a possible line of attack. Consider the superlative sentence in (45) below, where possible appears postnominally and where the superlative suffix is attached to the amount adjective few.

(45) I talked to the fewest guests possible.

At first sight, this case does not seem interestingly different from those presented above. In accordance with Larson's proposal, this example can receive the paraphrase in (46), where possible combines with an overt infinitival clause into a reduced relative.

(46) I talked to the fewest guests [possible for me to talk to]. 
What makes this case special, however, is that the reduced relative clause cannot be considered a restrictive relative clause of the usual sort. The sentences in (45) and (46) do not seem to make reference to the set of all groups of guests that it was possible for me to talk to; they cannot be paraphrased by saying that I talked to the smallest group in that set. Instead, they convey that I talked to as few guests as I could. This equative paraphrase suggests that the relative clause in these cases does not abstract over individuals, but instead abstracts over degrees.

This invites the speculation that the relative clauses in (45) and (46) are to be analyzed as so-called degree relatives of the sort first discussed in Carlson (1977). More generally, I speculate that postnominal possible is always interpreted as being part of a reduced degree relative. This may then open the way for an explanation of the equivalence of postnominal possible and prenominal non-local possible in superlatives. Future work will have to show whether this speculation can be extended into a concrete analysis.

\section{Concluding Remarks}

To summarize again, the analysis of modal superlatives presented here derives non-local readings for modal superlatives by positing a modal degree operator est possible and logical forms analogous to those for comparative readings in the analysis of Heim (1999). It is mainly motivated by agreement sharing facts in German and related restrictions on modal superlatives in English, but also by observations suggesting that the modal degree operator is subject to the same restrictions on covert movement as bare est.

On the downside, we have seen that the semantics of the modal degree operator cannot be compositionally derived and that there are empirical questions concerning uniqueness implications. In addition, it is apparent that the modal degree operator analysis is much less general than the implicit relative clause analysis proposed in Larson (2000). It remains to be seen how the modal degree operator analysis can be reconciled with the equivalence of modal superlatives with prenominal and postnominal possible. Another obvious open issue in the present proposal is the analysis of non-local possible in universals. The modal degree operator analysis remains silent on these cases while the implicit relative analysis extends to them naturally.

These results suggest that neither of the two analyses discussed in this paper is completely on the mark and that the proper analysis of modal superlatives is yet to be found. In fact, there are certain modal superlatives whose interpretation challenges both analyses under consideration in this paper. For example, it seems that neither of the two analyses derives the perceived interpretation of sentence (47). This sentence does not convey that this is the best answer it can be but rather that it is the best answer that can be given. ${ }^{9}$

This is the best possible answer. 
This reading cannot be credited to a local construal of possible because it is preserved in (48), where possible is postnominal and also because the German equivalent of (47) features agreement sharing. It is evident, therefore, that neither of the two analyses considered here has the intended effect in this particular case.

This is the best answer possible.

Examples of this kind suggest that in their present form, both the implicit relative analysis and the modal degree operator analysis are too rigid to account for the full range of non-local interpretations actually attested. Exploring the implication of this observation is another urgent task for future work on this topic.

\section{Endnotes}

* For comments I thank audiences at the University of Massachusetts at Amherst, the University of Calgary, McGill University, and Salt XV. Thanks for their time especially to Rajesh Bhatt, Irene Heim, Angelika Kratzer, and Junko Shimoyama. ${ }^{1}$ These reduced relatives are unusual in that the relativized position is a nonsubject. Run-of-the-mill reduced relatives (as in a book [stolen from the library]) are always subject relatives. Larson does not comment on this interesting aspect of the analysis.

${ }^{2}$ This analysis assumes that a finite clause can license ellipsis of an infinitival clause. The reader is referred to Larson's paper for a defense of this assumption.

${ }^{3}$ Given that the relative pronoun is silent, it is of course also conceivable that the entire relative clause is promoted to prenominal position.

${ }^{4}$ The present analysis is in danger of overgenerating, though. If est possible has the same categorial status as est, one should expect it to be able to attach to adjectives other than those that modify common nouns. An internet search produced examples like I ran the fastest possible and $F$ ast food joints will show the best possible looking sample of their product, but such cases are rare and perhaps marginal.

${ }^{5}$ For discussion and alternative analyses of comparative superlatives, see also Gawron (1995), Farkas and Kiss (2000), and Sharvit and Stateva (2002).

${ }^{6}$ Heim assumes that the value of $\mathrm{C}$ can in part be determined by focus and shows how this can be derived within Rooth's (1992) theory of focus. The intended value for $\mathrm{C}$ in (28) can result from focus marking on the subject John. In fact, stress on John seems to help bringing out the comparative reading.

${ }^{7}$ At least some speakers also accept I did my job as best I could, where it definitely appears that either $a s$ or the superlative morphology cannot have its ordinary meaning.

${ }^{8}$ Building on discussion in Heim (1999), Sharvit and Stateva (2002) propose that the absolute/comparative distinction is a case of pragmatics context dependency rather that a genuine ambiguity of logical form. However, the observation that the 
availability of comparative readings is subject to structural constraints seems to argue against a purely pragmatic account.

${ }^{9}$ I thank Brendan Gillon and Alan Bale for discussion of related cases.

\section{References}

Abney, Stephen: 1987, The English Noun Phrase in Its Sentential Aspect, Ph.D. dissertation, MIT.

Bresnan, Joan: 1973, 'Syntax of the comparative clause construction in English', Linguistic Inquiry 4, 275-343.

Carlson, Greg: 1977, 'Amount Relatives', Language 53, 520-542.

Corver, Norbert: 1997, 'Much-support as last resort', Linguistic Inquiry 21, 119 164.

Farkas, Donka F. and Katalin E. Kiss: 2000, 'On the Comparative and Absolute Reading of Superlatives', Natural Language and Linguistic Theory 18, 417-455.

Gawron, Jean M.: 1995, 'Comparatives, superlatives, and. resolution', Linguistics and Philosophy 18, $333-380$.

Heim, Irene: 1985, 'Notes on Comparatives and Related Matters', Ms, The University of Texas at Austin.

Heim, Irene: 1999, 'Notes on Superlatives', Ms, MIT.

Heim, Irene: 2000. 'Degree Operators and Scope', in B. Jackson and T. Matthews (eds.), Proceedings of Semantics and Linguistic Theory $X$, CLC Publication, Cornell University, Ithaca.

Larson, Richard: 2000, 'ACD in AP?', paper presented at the 19th West Coast Conference of Formal Linguistics, Los Angeles, CA.

May, Robert: 1985, Logical From: Its Structure and Derivation, MIT Press, Cambridge.

Rooth, Mats: 1985, Association with Focus, Ph.D. dissertation, University of Massachusetts at Amherst.

Sag, Ivan: 1976, Deletion and Logical Form, Ph.D. dissertation, MIT. Published 1980 by Garland, New York.

Sharvit, Yael and Penka Stateva: 2002, 'Superlative expressions, context, and focus', Linguistics and Philosophy 23, 453-504.

Szabolcsi, Anna: 1986, 'Comparative Superlatives', in N. Fukui et al. (ed.), MIT Working Papers in Linguistics, Vol. 8, MIT Press, Cambridge, pp. 245265. 